\title{
The effect of rotenone on the dopaminergic cells of the substantia nigra of the midbrain in the adult male albino rats
}

\author{
MOHAMMED ABDEL-RAHMAN ${ }^{1}$, RANIA A. GALHOM ${ }^{2}$, WAEL AMIN NASR \\ EL-DIN $^{2,4}$, MONA H. MOHAMMED ALI ${ }^{2}$, ALAA EL-DIN SAAD ABDEL- \\ HAMID $^{3}$
}

\begin{abstract}
${ }^{I}$ Department of Anatomy \& Embryology, Faculty of Medicine, Sohag University, Sohag, Egypt, ${ }^{2}$ Department of Anatomy \& Embryology, Faculty of Medicine, Suez Canal University, Ismailia, Egypt, ${ }^{3}$ Department of Clinical Pathology, Faculty of Medicine Suez Canal University, Ismailia, Egypt,

${ }^{4}$ Department of Anatomy, Ibn Sina National College for Medical Studies, Jeddah, Saudi Arabia.
\end{abstract}

\begin{abstract}
Background aims. Rotenone is a widely used insecticide has a neurodegenerative effect on the dopaminergic cells of substantia nigra (SN) of midbrain producing Parkinsonism. The aim of this study is to study the effects of rotenone when injected subcutaneously on the dopaminergic cells of the substantia nigra of the midbrain. Methods. The effects of rotenone on the SN of midbrain and the were determined histopathologically, immunohistochemically, and by transmission electron microscopy. Results. Subcutaneous rotenone produced Parkinsonism through producing degeneration of the dopaminergic cells of SN of the midbrain. Conclusion. These results indicate that, rotenone has a neurodegenerative effect on the dopaminergic cells of the substantia nigra of the midbrain producing parkinsonism.
\end{abstract}

Key words: Dopaminergic neurons, neurodegeneration, Parkinsonism, rotenone, Substantia nigra.

\section{Introduction}

Rotenone produces its cytotoxic effects by interfering with the electron transport chain in mitochondria. This interferes with $\mathrm{NADH}$ during the creation of usable cellular energy (ATP) leading to oxidative stress and the cellular oxygen is reduced to produce a reactive oxygen species which destroy the cellular DNA and mitochondria [3]. It can easily affects the central nervous system and other body systems because it is highly lipophilic, so, it crosses the blood brain barrier and cellular membrane easily affecting the nervous system [6].

Rotenone is a potential cause of Parkinsonism because the long-term exposure to rotenone causes cellular changes in the form of injury to central dopaminergic neurons, degeneration and apoptosis of substantia nigra dopaminergic neurons and the
Rotenone is an effective natural broad spectrum pesticide and insecticide [1] that is extracted from the roots of certain tropical and subtropical legume plants and widely used all over the world since decades. Its use in agriculture can cause serious pollution of streams and reservoirs since it can easily reach the soil with heavy rains [2]. The degradation can sometimes persist for months according to a variety of factors including light, temperature, depth in the soil, dose and the presence of organic debris, for example decomposition occurs faster as the temperature of the water is higher [3].

It is often formulated as dusts, powders and sprays for use in gardens and on food crops [4]. It is whitish in color and odorless [5]. 
important mechanism leading to mitochondrial and neuronal dysfunction and degeneration [19]. Also, there are evidences that Parkinsonism is related to mitochondrial dysfunction through reducing mitochondrial complex activity as this leads to neuronal degeneration by decreasing energy production, so Parkinsonism can be produced by any cause of mitochondrial dysfunction including pesticides [20].

\section{Material and methods}

This work was achieved in Ain Shams Hospitals, Cairo, Egypt. Experimental and physiological assessment was performed in Suez Canal University, Ismailia, Egypt.

\section{Induction of Parkinsonism}

Parkinsonism was induced by rotenone (Sigma-Aldrich, St. Louis, MO, USA). Rotenone was dissolved in dimethyl sulfoxide (DMSO) (Sigma-Aldrich, St. Louis, MO, USA) and injected subcutaneously in a dose of $\mathbf{1 . 5} \mathrm{mg} / \mathrm{kg}$ body weight every forty eight hours for six doses [21].

\section{Evaluation of motor function and balance}

To assess the balance and motor coordination, each rat was placed on a horizontal rotating rod $(\mathbf{1 0} \mathrm{cm}$ long and $4 \mathrm{~cm}$ in diameter). The rod rotation speed was $20 \mathrm{rpm}$. Each rat was left on the rotating rod and the time spent from putting the animal on the shaft of the rota-rod, till it falls to the ground (Latency) was recorded. The maximal time that was allowed for each rat was 5 minutes [22].

\section{Histopathological evaluation of midbrain}

Haematoxylin and eosin; for morphological assessment [23] and Masson-Fontana method; for the detection and evaluation of the dopaminergic melanin pigment producing cells in the $\mathrm{SN}$ of midbrain [24] were performed. formation of Lewy bodies in neurons indicating neurodegenerative effects [7].

Parkinsonism is the second most prevalent neurodegenerative disease after Alzheimer's disease [8] and the first most common motor neurodegenerative disease [9] affecting about $1-3 \%$ of the population over 50 years of age and $5 \%$ of the population over 65 [10] as its incidence and severity increases with age [11] and consistently affects males more than females and has a genetic susceptibility leading to familial and sporadic cases of Parkinsonism [12]. Also it was evidenced that Parkinsonism is more common in diabetics than nondiabetics and more in cases of uncontrolled diabetes than whose diabetes is well managed and controlled because diabetes helps the damage of nerve cells while the antidiabetic drugs suppress the progression of Parkinsonism through the inhibition of microglial activation [13]. In addition, Parkinsonism is $12 \%$ higher in urban than rural areas [14] and is heritable only in approximately $\mathbf{5 \%}$ of familial cases [15].

Parkinsonism is characterized by death of the dopaminergic neurons in substantia nigra pars compacta [16]. Degeneration of pigmented neurons in this region is the principal pathology that

underlies Parkinsonism. In a few people, the cause of Parkinsonism is genetic but in most cases the reason for the death of these dopamine neurons is unknown [17].

The neuronal degeneration of dopaminergic cells is as a result of some cellular changes including oxidative stress, mitochondrial dysfunction, inflammation and atypical apoptosis [18]. There are evidences demonstrate that an altered balance in mitochondrial division and fusion is an 
Electron microscopy

Ultrathin sections were examined using a TEM 10 transmission electron microscope (Zeiss, Jena, Germany) to evaluate the ultrastructural changes of the dopaminergic cells of the substantia nigra $[\mathbf{2 7}, \mathbf{2 8}]$.
Tyrosine hydroxylase

(TH) immunohistochemistry

$\mathrm{TH}$ is a cytoplasmic immunohistochemistry stain was used to evaluate the $\mathrm{TH}+$ dopaminergic neurons in the $\mathrm{SN}[\mathbf{2 5}, \mathbf{2 6}]$ and their degeneration as a result of exposure to rotenone.

\section{Results}

Histopathological examination:

A) Results of hematoxylin and eosin and Masson-Fontana stained sections:

In the control group, the SN of midbrain appeared normal with the presence of the characteristic densely packed dopaminergic cells (Figure $1 \mathbf{a}$ ), while in the group exposed to rotenone, there was marked reduction in the number of dopaminergic cells in the SN with numerous vacuoles (Figure 1 b).

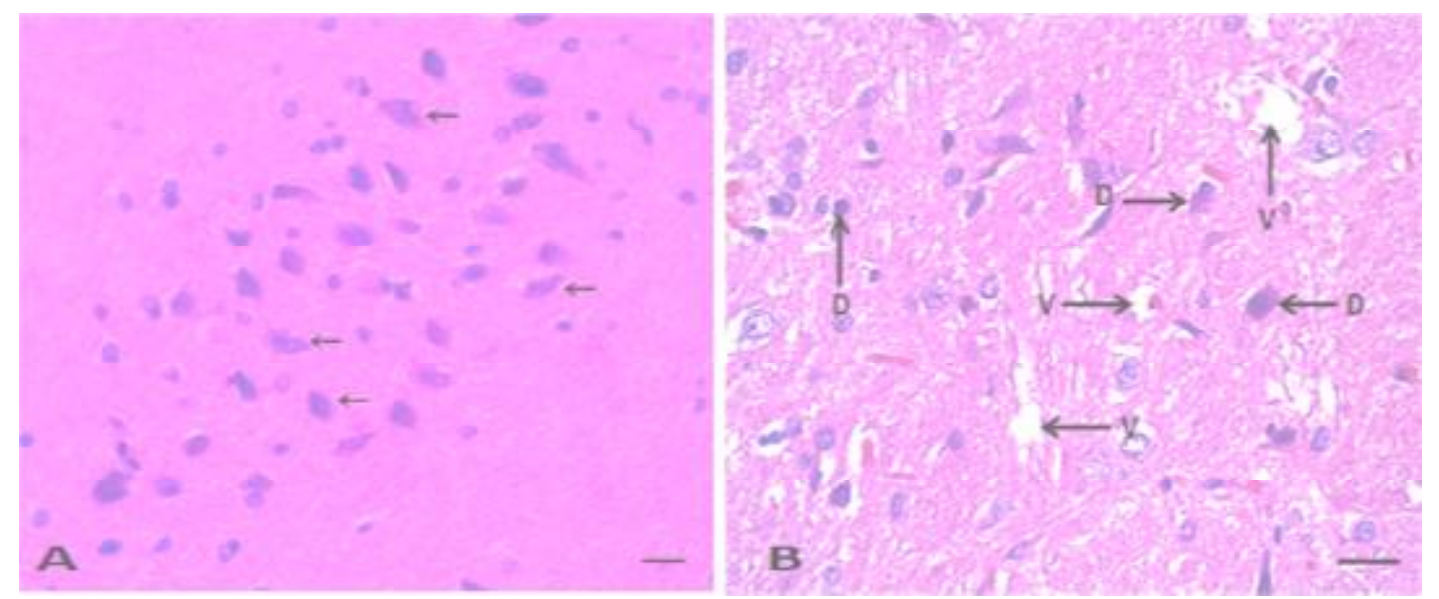

Figure 1. Photomicrographs of midbrain sections of male albino rats stained with hematoxylin \&

eosin showing normal structure with dopaminergic cells (arrows) containing melanin pigment in the $\mathrm{SN}$ in the control group (A), while in the group exposed to rotenone (B), there is decreased number of dopaminergic cells [D] and the appearance of degenerative vacuoles [V]. Scale bar $20 \mu \mathrm{m}$. X 400.

\section{B) Results of Masson-Fontana stained sections:}

In the control group, the SN of midbrain appeared normal with the presence of the characteristic densely packed dopaminergic cells (Figure $\mathbf{2}$ a), while in the group exposed to rotenone, there was marked reduction in the number of dopaminergic cells in the $\mathrm{SN}$ with numerous vacuoles (Figure $\mathbf{2}$ b). 


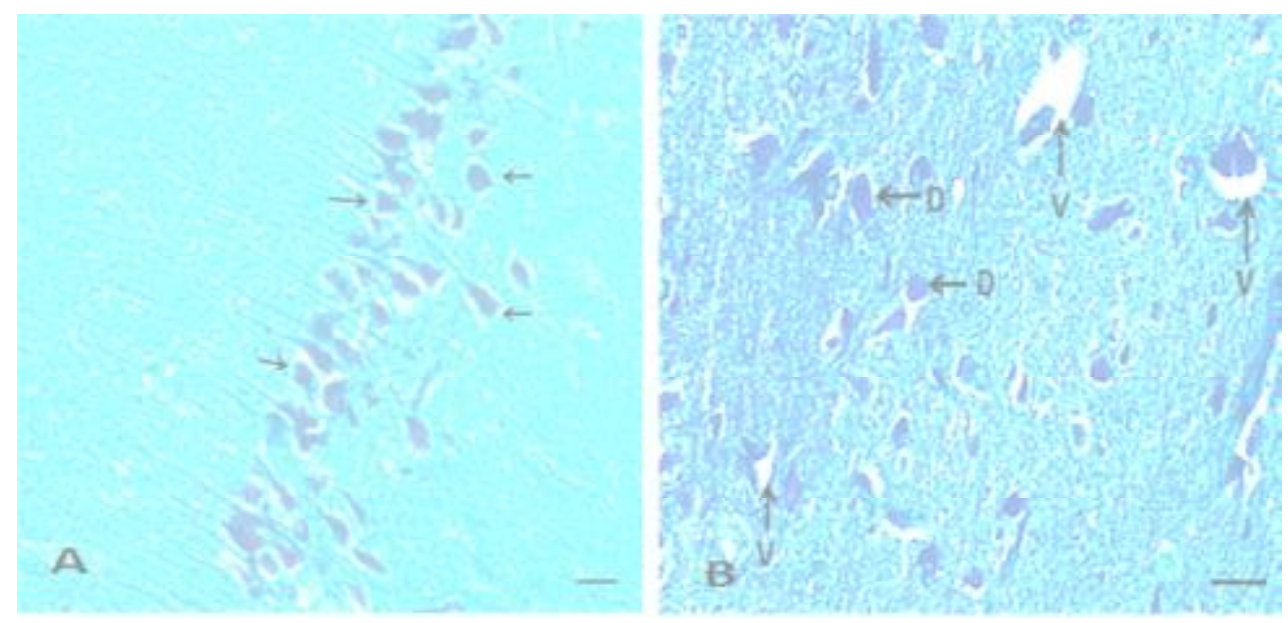

Figure 2. Photomicrographs of midbrain sections of male albino rats stained with Masson Fontana

showing normal structure with dopaminergic cells (arrows) containing melanin pigment in the $\mathrm{SN}$ in

the control group (A), while in the group exposed to rotenone(B), there is decreased number of dopaminergic cells [D] and the appearance of degenerative vacuoles [V]. Scale bar $40 \mu \mathrm{m}$. X 200.

\section{C) Results of tyrosin hydroxylase (TH) immunohistochemistry:}

In the control group, the $\mathrm{SN}$ showed normal appearance with large number of $\mathrm{TH}$ positive dopaminergic cells identified by their dark brown color (Figure 3 a). In group treated with rotenone, there was obvious reduction in the number of the dopaminergic cells in the $\mathrm{SN}$ (Figure $3 \mathbf{b}$ ).

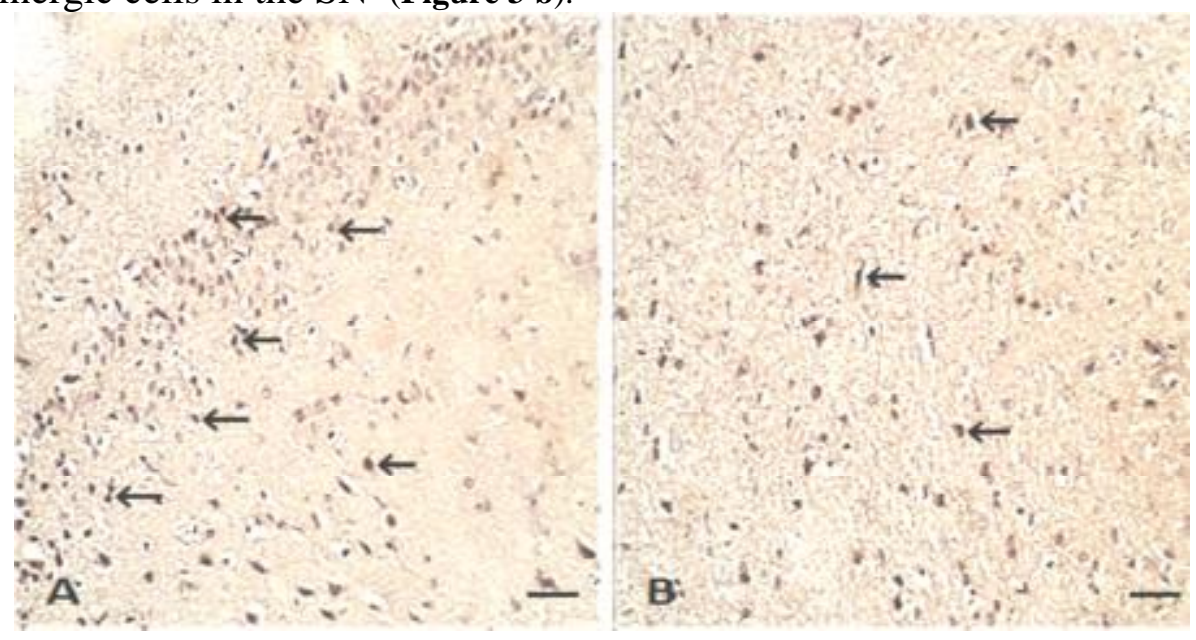

Figure 3. Photomicrographs of midbrain sections of adult male albino rats showing normal structure

with dopaminergic cells (arrows) containing melanin pigment with positive immunostaining of $\mathrm{TH}$ in the $\mathrm{SN}$ in the control group (A), while in the rotenone group (B), there is decreased number of dopaminergic cells (arrows). Scale bar $\mathbf{1 0 0} \mu \mathrm{m}$. (TH immunostain, $\mathrm{x}$ 100).

\section{D) Results of transmission electron microscopy (TEM):}

In the control group, the $\mathrm{SN}$ showed normal mitochondria with normal membranes and cristae and normal nuclear morphology with dispersed chromatin. Also, the 
myelin sheaths around the axons appeared normal i.e. thick, regular and continuous (Figure 4 a). In the rotenone group, there was degeneration of the dopaminergic cells of $\mathrm{SN}$ in the form of mitochondrial distension and ruptured cisterns. The cytoplasm showed vacuoles. Also there were thinned interrupted myelin sheaths around the axons (Figure 4 b).
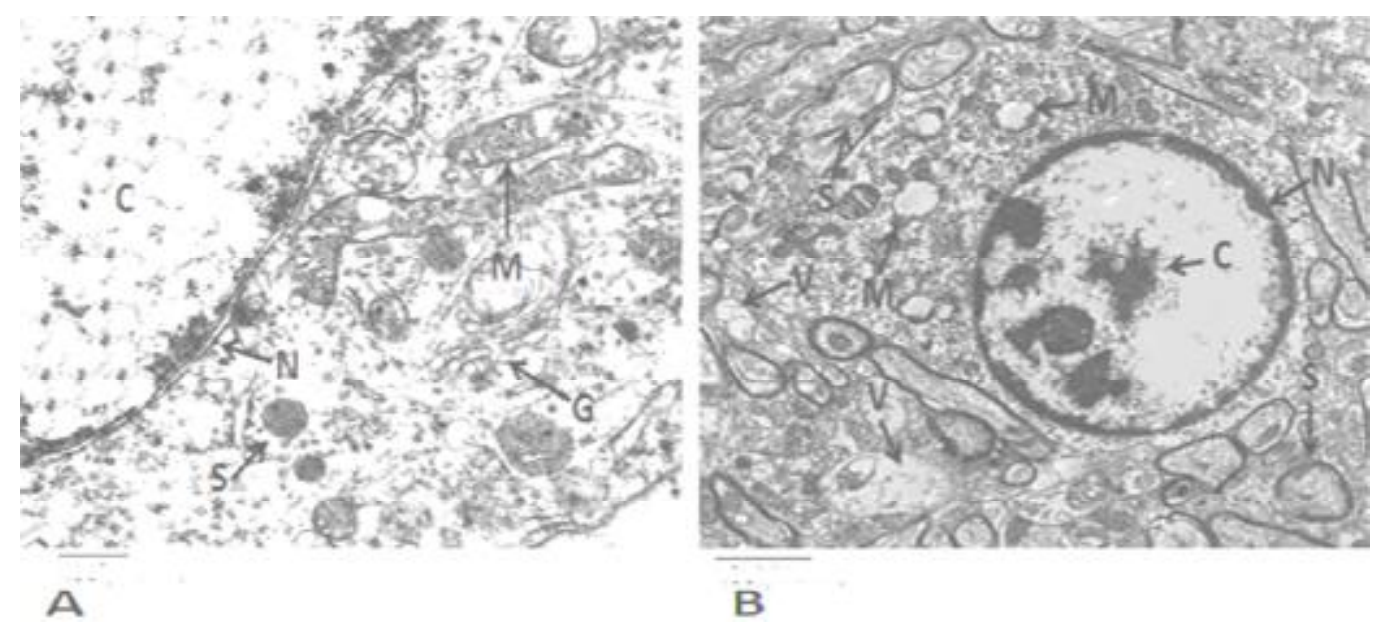

B

Figure 4. Transmission electron micrographs of the midbrain of male albino rats showing a

dopaminergic cell with normal nucleus $[\mathrm{N}]$, nuclear homogenously distributed chromatin [C], normal mitochondria [M], Golgi apparatus [G] and normal myelin sheaths $[\mathrm{S}]$ i.e. thick, regular and continuous in the control group micrograph (A). Scale bar $500 \mu \mathrm{m}$. X 30000. While the rotenone group micrographs (B) showing degenerative changes; condensate nuclear chromatin $[\mathrm{C}]$ in the center of the nucleus $[\mathrm{N}]$, degenerated mitochondria [M] (Distended with ruptured cisterns), numerous degenerative cytoplasmic vacuoles [V] and the myelin sheaths $[\mathrm{S}]$ are thin, irregular and discontinuous. Scale bar $2 \mu \mathrm{m}$. X $\mathbf{1 0 0 0 0 .}$

\section{Motor evaluation (Rota rod test)}

The motor balance and motor coordination were evaluated by rota-rod test in which each rat was placed on a horizontal rotating rod and the latency (Time in seconds spent from putting the animal on the rotating rod till it falls to the ground) was recorded. The results indicated highly significant drop of the rota-rod test in group B (The rotenone group) compared to group A (The control group) indicating motor disturbances of Parkinsonism induced by rotenone. In group $\mathrm{C}$ (The OSCs-treated group) there was a marked motor improvement when compared to group B indicating the ability of OSCs to reach the substantia nigra of midbrain to replace the dopaminergic cells damaged by rotenone as shown in table $\mathbf{1} \&$ figure 5. 
Table 1: Mean \pm SD of the rota-rod test of the three groups

\begin{tabular}{|l|l|l|}
\hline Group & Mean & Std. Deviation \\
\hline Group A (Control Group) & 63.9000 & 9.96048 \\
\hline Group B (Rotenone Group) & 20.6000 & 6.96340 \\
\hline Group C (OSCs-treated group) & $\mathbf{4 6 . 1 0 0 0}$ & $\mathbf{6 . 3 3 2 4 6}$ \\
\hline Total & $\mathbf{4 3 . 5 3 3 3}$ & $\mathbf{1 9 . 6 2 0 0 7}$ \\
\hline
\end{tabular}

ANOVA $=\mathbf{7 5 . 6 6 5}, \mathrm{P}$ value $<\mathbf{0 . 0 0 1}(\mathrm{HS})$

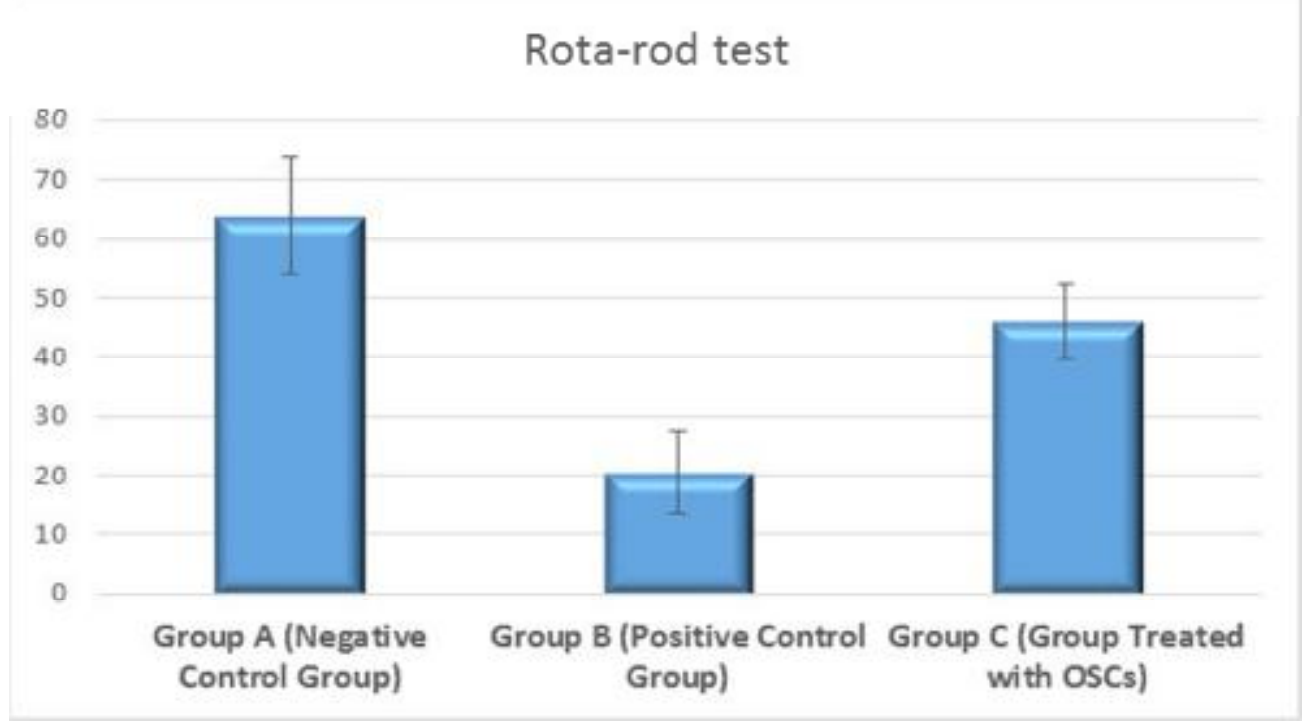

Figure 5. Mean \pm SD of the rota-rod test of the three groups.

\section{Discussion}

Parkinsonism is a common neurodegenerative disease resulting from degeneration of the dopaminergic cells of substantia nigra of midbrain and manifested by motor and psychological problems. To date, no curative treatment for Parkinsonism has been proven apart from certain drugs for only symptomatic relief. However, these available drugs have numerous side effects.

Substantia nigra injury has a very poor capacity for intrinsic regeneration. Problems such as limited cell sources, high incidence of the neurodegenerative disorders affecting substantia nigra and hazardous effects of long term therapeutic regimens result in the need for cell replacement therapy to treat these neurodegenerative diseases such as Parkinsonism [29].

In the current study, rotenone was used to induce Parkinsonism because rotenone is a widely used broad spectrum insecticide and pesticide and easily produced by extraction from the roots and stems of several tropical and subtropical plant species, especially those belonging to the genera Lonchocarpus and Derris [30]. Rotenone can be absorbed through the gastrointestinal mucosa if ingested orally [31] and adequately absorbed if injected subcutaneously then it can cross the blood brain barrier in sufficient amounts to produce oxidative stress toxic effects on the brain tissues mainly through mitochondrial destruction. This results in numerous neurodegenerative 
disorders including non-specific central nervous system toxicity resulting in induction of apoptosis and degeneration of the dopaminergic nerve cells of the substantia nigra of midbrain leading to Parkinsonism [32].

Rotenone is extremely hydrophobic in nature, so it can be given by any route. It crosses the biological membranes easily including the blood brain barrier and does not depend on the dopamine transporter for acquiring access to the cytoplasm of dopaminergic neurons [33].

In agreement with our study, Schuler and Casida, (2001) [34] detected that rotenone administration leads to the appearance of motor and none motor parkinsonian manifestations as it inhibits the activity of NADHubiquinone reductase leading to the formation of reactive species, such as superoxide anions, and the formation of these reactive species affects the dopaminergic cells of SNpc seriously because of the high levels of metabolism in substantia nigra, the high prevalence of glial cells, and particularly the low levels of antioxidant defenses in this region. Also, Pan et al. (2009) [35] have shown that rotenone induces neuronal degeneration through the induction of apoptosis and oxidative stress and it produces Parkinsonism through highly selective neurodegeneration of dopaminergic cells.

In our study, injection of rotenone into the rats resulted in degenerative effects on the substantia nigra of the midbrain specimens. These neurodegenerative changes were in the form of marked reduction in the number of dopaminergic cells. This was in accordance with Alam and Schmidt, (2002) [36] as they detected that, rotenone destroys dopaminergic neurons of SNpc leading to depletion of the neurotransmitter dopamine with appearance of the parkinsonian symptoms in rats. Also, Kim et al. (2009) [37] explained that, rotenone crosses the blood brain barrier to enter the nerve cells then, inside the nerve cells, rotenone inhibits mitochondrial respiration, thus elicits ATP deficiency, produces reactive oxygen species, and disrupts $\mathrm{Ca} 2+$ homeostasis, downstream mitochondrial damage leading to release of cytochrome $\mathrm{C}$ and caspase- 3 activation and finally causing apoptotic death.

The current ultrastructural findings exhibited disturbances in the nuclear chromatin that became fragmented instead of being homogenously distributed in rotenone treated group. This was in correlation with results of Mani et al. (2014) [33] who reported that, the pathological features of rotenone effects on substantia nigra dopaminergic cells include significantly increased number of cells showing apoptotic features in the form of rupture of the cell membrane and nuclear DNA damage (fragmentation of nuclear chromatin).

Another finding was mitochondrial degeneration in the form of swollen vacuolated mitochondria with loss of their cisternae. This is in agreement with the findings of Dauer and Przedborski, (2003) [6] who detected that pathogenesis of Parkinsonism includes oxidative stress in the form of mitochondrial dysfunction and degeneration, and with Cabezas et al. (2012) [38] who reported, the oxidative impairment of mitochondrial function induced by rotenone causes bioenergetic failure through the production of oxidative molecules causing mitochondrial damage and cell death accompanied by microglial activation and neuroinflammation.

It has been mentioned that, Parkinsonism results from the degeneration of dopamine-producing nerve cells in the substantia nigra of 
the midbrain and the clinical signs of Parkinsonism appear when about $70 \%$ of the dopamine-producing neurons are damaged [16]. Moreover, RodriguezOroz et al. (2009) [39] detected that Parkinsonism is associated with the selective loss of dopaminergic neurons in the SNpc leading to decreased dopamine level in the corpus striatum because dopamine reached the corpus striatum through the nigrostriatal dopamine pathway in the brain, this causes de-regulation in the basal ganglia circuits that leads to the appearance of motor symptoms such as bradykinesia, resting tremor, rigidity, and postural instability as well as nonmotor symptoms such as sleep disturbances, depression, and cognitive deficits.

The known ultrastructural changes in the dopaminergic neurons undergoing neurodegeneration in cases of Parkinsonism including mitochondrial changes such as swelling, vacuolations, distorted cristae and empty mitochondria due to oxidative mitochondrial stress and nuclear disturbance such as shrunken nuclei and condensation of the nuclear chromatin [40] are in correlation with our findings concerning the group treated with rotenone.

Concerning the functional motor evaluation using the open field test and rota-rod test, the current findings were in agreement with the results of many previous studies such as those done by Fleming et al. (2013) [22] as they used the rota-rod test to evaluate the motor disturbances of Parkinsonism.

As a conclusion of the current study, rotenone that is a widely used insecticide and pesticide has a neurodegenerative effect on the dopamine producing cells of the substantia nigra of the midbrain leading to Parkinsonism.

\section{References}

1. Wood DM, Alsahaf $H$, Streete $P$, Dargan PI, and Jones AL (2005): Fatality after deliberate ingestion of the pesticide rotenone: a case report. Crit Care; 9(3):280-284.

2. Tanner CM, Kamel F, Ross GW, Hoppin JA, Goldman SM, Korell M; Marras C, Bhudhikanok GS, Kasten M, Chade AR, Comyns K, Richards MB, Meng C, Priestley B, Fernandez HH, Cambi F, Umbach DB, Blair A, Sandler DP and Langston JW. (2011): Rotenone, Paraquat and Parkinson's Disease. Environmental Health Perspectives; 119 (6): 866872.

3. Markham A, Cameron I and Bains R. (2012): Brain-derived neurotrophic factor-mediated effects on mitochondrial respiratory coupling and neuroprotection share the same molecular signalling pathways. Eur J Neurosci; 35:366-374.

4. Ren Y and Feng J. (2007): Rotenone selectively kills serotonergic neurons through a microtubule-dependent mechanism. J Neurochem; 103:303311.

5. Dhaouadi A, Monser L and Adhoum N. (2010): Removal of rotenone insecticide by adsorption onto chemically modified activated carbons. J Hazard Mater; 181:692699.

6. Dauer $\mathrm{W}$ and Przedborski S. (2003): Parkinson's disease: mechanisms and models. Neuron; 39 (6):889-909.

7. Gomez C, Bandez MJ and Navarro A. (2007): Pesticides and impairment of mitochondrial function in relation with the parkinsonian syndrome. Front Biosci; 12:10791093.

8. Wirdefeldt $\mathrm{K}$, Adami $\mathrm{HO}$, Cole $\mathrm{P}$, Trichopoulos D and Mandel J. 
(2011): Epidemiology and etiology of Parkinson's disease: a review of the evidence. Eur J Epidemiol; 26:1-58.

9. Jankovic J. (2008): Parkinson's disease: clinical features and diagnosis. J Neurol Neurosurg Psych; 79:368-376.

10. Alves G, Forsaa EB, Pedersen KF, Dreetz Gjerstad, and Larsen M. (2008): Epidemiology of Parkinson's disease. J Neurol; 255:18-32.

11. Joers V and Emborg M. (2009): Preclinical assessment of stem cell therapies for neurological diseases. ILAR J; 51:24-41.

12. Karin W, Hans-Olov A, Philip C, Dimitrios $T$ and Jack M. (2011): Epidemiology and etiology of Parkinson's disease: a review of the evidence. Eur J Epidemiol; 26:1-58.

13. Sadeghian M, Marinova-Mutafchieva L, Broom L, Davis J, Virley D and Medhurst A. (2012): Full and partial peroxisome proliferation-activated receptor-gamma agonists, but not delta agonist, rescue of dopaminergic neurons in the 6-OHDA parkinsonian model is associated with inhibition of microglial activation and MMP expression. J Neuroimmunol; 246:69-77.

14. Horsfall L, Petersen I, Walters $\mathrm{K}$ and Schrag A. (2013): Time trends in incidence of Parkinson's disease diagnosis in UK primary care. $\mathrm{J}$ Neurol; 260:1351-1357.

15. Lesage $S$ and Brice A. (2009): Parkinson's disease: From monogenic forms to genetic susceptibility factors. Hum Mol Genet; 18:48-59.

16. Kim S, Sung J, Um J, Hattori N, Mizuno Y, Tanaka K, Paik S, Kim J and Chung K. (2003): Parkin cleaves intracellular synuclein inclusions via the activation of calpain. $J$ Biol Chem; 278:41890-41899.
17. Sasaki M, Shibata E, Tohyama K, Takahashi J, Otsuka K, Tsuchiya K, Takahashi S, Ehara S, Terayama Y and Sakai A. (2006): Neuromelanin magnetic resonance imaging of locus ceruleus and substantia nigra in Parkinson's Neurorep; 17:1215-1218.

18. Sami M, Kumar M, Kumar A, Shukla $S$ and Pratap M. (2015): Resveratrol protects from toxin-induced Parkinsonism. Mol Neurobiol; 5:110.

19. Xinglong W, Timothy G, Yingchao L, Jun L, Hisashi F and Xiongwei Z. (2012): Parkinson's disease associated DJ-1 mutations impair mitochondrial dynamics and cause mitochondrial dysfunction. J Neurochem; 121:830-839.

20. Terina N, Martinez J and Timothy G. (2012): Toxin models of mitochondrial dysfunction in Parkinson's disease. Antioxidants \& Redox Signaling; 16:920-934.

21. Zaitone SA, Abo- Elmatty DM and Elshazly SM. (2012):Piracetam and vinpocetine ameliorate rotenone induced Parkinsonism in rats. Indian J Pharmacol; 44:774-779.

22. Fleming SM, Ekhator OR ang Ghisays V. (2013): Assessment of Sensorimotor Function in Mouse Models of Parkinson's Disease. J Vis Exp; 76:50303.

23. Budde MD, Kim JH, Liang HF, Schmidt RE, Russell JH and Cross AH. (2007): Toward accurate diagnosis of white matter pathology using diffusion tensor imaging. Magn Reson Med; 57:688-695.

24. Bishop JA, Nelson AM, Merz WG, Askin FB and Riedel S. (2012): Evaluation of the detection of melanin by the Fontana-Masson silver stain in tissue with a wide 
range of organisms

including Cryptococcus. Human Pathol; 43:898-903.

25. Jiang J, Jiang J, Zuo $\mathrm{Y}$ and $\mathrm{Gu} \mathrm{Z}$. (2013): Rapamycin protects the mitochondria against oxidative stress and apoptosis in a rat model of Parkinson's disease. Int J Mol Med; 31:825-832.

26. Yilmazer-Hanke D, Eliava M, Hanke J, Schwegler H and Asan E. (2016): Density of acetylcholine esterase (AchE) and tyrosine hydroxylase (TH) containing fibers in the amygdala of roman high-and lowavoidance rats. Neurosc Lett; 632:114-118.

27. Lennington JB, Pope S, Goodheart AE, Drozdowicz L, Daniels SB and Salamone JD. (2011): Midbrain dopamine neurons associated with reward processing innervate the neurogenic subventricular zone. J Neurosci; 31:13078-13087.

28. Watabe-Uchida M, Zhu L, Ogawa SK, amanrao $\mathrm{A}$ and Uchida $\mathrm{N}$. (2012): Whole-Brain Mapping of Direct Inputs to Midbrain Dopamine Neurons. Neuron; 74:858-873.

29. Olle L and Anders B. (2004): Cell Therapy in Parkinson's disease. Neuro; 1(4): 382-393.

30. Wrangel CV, Schwabe K, John N, Krauss JK and Alam M. (2015): The rotenone-induced rat model of Parkinson's disease: Behavioral and electrophysiological findings. Behav Br Res; 279:52-61.

31. Wood DM, Alsahaf $H$, Streete $P$, Dargan PI, and Jones AL (2005): Fatality after deliberate ingestion of the pesticide rotenone: a case report. Crit Care; 9(3):280-284.

32. Lapointe $\mathrm{N}$, St-Hilaire M, Martinoli MG, Blanchet J, Gould P, Rouillard $\mathrm{C}$ and Cicchetti F. (2004): Rotenone induces non-specific central nervous system and systemic toxicity. FASEB J; 18(6):717-719.
33. Mani $\mathrm{K}$,

Thamilarasan M,

Musthafa ME,

Kuppusamy T,

Govindasamy PS, Subran K and Justin AT (2014): Mangiferin Antagonizes Rotenone: Induced Apoptosis Through Attenuating Mitochondrial Dysfunction and Oxidative Stress in SK-N-SH Neuroblastoma Cells. Neurochem Res; 39(4):668-676.

34. Schuler F and Casida JE (2001): Functional coupling of PSST and ND1 subunits in NADH: ubiquinone oxidoreductase established by photo affinity labeling. Biochem Biophys Acta; 1506:79-87.

35. Pan T, Rawal P, Wu Y, Xie W, Jankovic $J$ and Le W. (2009): Rapamycin protects against rotenoneinduced apoptosis through autophagy induction. Neurosci; 164: 541-551.

36. Alam A and Schmidt WJ (2002): Rotenone destroys dopaminergic neurons and induces parkinsonian symptoms in rats. Behav Brain Res; 136:317-324.

37. Kim H, Park H, Park H and Chung J. (2009): Tranexamic acid protects against rotenone induced apoptosis in human neuroblastoma SH-SY5Y cells. Toxicol; 262:171-174.

38. Cabezas R, El-Bachá RS, Gonzáles J and Barreto GE (2012): Mitochondrial functions in astrocytes: neuroprotective implications from oxidative damage by rotenone. Neurosci Res; 74:80-90.

39. Rodriguez-Oroz MC, Jahanshahi M, Krack P, Litvan I, Macias $\mathrm{R}$ and Bezard E. (2009): Initial clinical manifestations of Parkinson's disease: features and pathophysiological mechanisms. Lancet Neurol; 8:11281139.

40. Jianhua J, Juean J, Yuanyi $\mathrm{Z}$ and Zhenlun G. (2013): Rapamycin protects the mitochondria against oxidative stress and apoptosis in a rat 
model of Parkinson's disease. Int $\mathrm{J}$ Mol Med; 31:825-832.

41. Mandillo S, Heise I, Garbugino L, Tocchini-Valentini GP, Giuliani A, Wells S and Nolan PM. (2014): Early motor deficits in mouse disease models are reliably uncovered using an automated home-cage wheelrunning system: a cross-laboratory validation. Disease models an mechanisms; 7:397-407. 